\title{
CONTINUOUS FUNCTIONS DEFINED ON PRODUCT-SPACES
}

\author{
WOLFGANG M. SCHMIDT
}

1. The results. The most concrete result of this paper is

TheOREM 1. Let $f(x, y)$ be a continuous double-periodic function satisfying $f(x+1, y)=f(x, y+1)=f(x, y)$. Let $\alpha, \beta$ be arbitrary. Then there exist $x, y, \bar{y}$ having

$$
f(x, y)=f(x, y+\beta)=f(x+\alpha, \bar{y})=f(x+\alpha, \bar{y}+\beta) .
$$

Thus $f$ maps the vertices of a certain parallelogram into a single number. In [1] I proved the theorem in special cases and showed that it has an application to continuous functions on the 3-sphere. In [1] I also showed that the theorem would no longer be true if one would ask for $y=\bar{y}$.

More generally, we say a class $\sigma_{k}$ of $k$-tuples of points in a compact topological space $S$ has property $p$, if every real-valued map $f$ of $S$ maps all the points of a $k$-tuple $\Sigma_{f} \in \sigma_{k}$ into a single point. Here and throughout the paper, compact means sequentially compact. Thus if $S$ is the $n$-sphere $S^{n}$ and $\sigma_{n+1}$ the class of orthogonal $(n+1)$-tuples on $S$, then the Kakutani-Yamabe-Yujobo-Theorem states that $\sigma_{n+1}$ has property $p$.

We call the topological product of a line with $S$ cylinder over $S$ and denote it by $C(S)$. Points of $C(S)$ will be written $(x, X)$, where $x$ is a real number, $X \in S$. A continuous curve in $C(S), x(t), X(t),-\infty<t$ $<\infty$, will be called a rain over $S$ if $x(t)$ tends to $\pm \infty$ when $t$ tends to $\pm \infty$. A roof over $S$ is a compact set in $C(S)$ which has a nonempty intersection with every rain over $S$. A class $\sigma_{k}$ of $k$-tuples in $S$ has property $P$ if to any roof $R$ over $S$ there exists a $k$-tuple $\Sigma_{R} \in \sigma_{k}$ and an $x$ such that

$$
(x, X) \in R \text { for every } X \in \Sigma_{R} .
$$

Since every real-valued map $f$ of a compact space $S$ is associated with the roof $(f(X), X)$, property $P$ implies $p$.

Now let $X_{1}, \cdots, X_{n+1}$ be an $(n+1)$-tuple of points on the $n$ sphere $S^{n}$ whose spherical distances satisfy

$$
d\left(X_{i}, X_{j}\right)=d\left(X_{1}, X_{j}\right) \quad(1 \leqq i<j \leqq n+1) .
$$

Let $\tau_{n+1}$ be the class of $(n+1)$-tuples obtained by applying a rotation

Received by the editors December 13, 1960. 
to our particular $X_{1}, \cdots, X_{n+1}$. Then the methods of YamabeYujobo [2] show that $\tau_{n+1}$ has property $P$.

We say a sequence $\Sigma_{1}, \Sigma_{2}, \cdots$ of $k$-tuples is convergent to a $k$-tuple $\Sigma$, if the elements $X_{i 1}, X_{i 2}, \cdots, X_{i k}$ of $\Sigma_{i}$ and $X_{1}, \cdots, X_{k}$ of $\Sigma$ can be arranged in such a way that $\lim X_{i j}=X_{j}(j=1, \cdots, k)$. We call a class $\sigma_{k}$ closed if the limit of any convergent sequence of $k$ tuples of $\sigma_{k}$ is again in $\sigma_{k}$.

If $\sigma_{k}$ is a class of $k$-tuples in $S$ and $\tau_{l}$ a class of $l$-tuples in $T$, then we define $\sigma \times \tau$ to be the class of the following $k \cdot l$-tuples in the topological product $S \times T$. The $k \cdot l$-tuples of $\sigma \times \tau$ consist of all pairs of the type $(X, Y)$, where $X$ runs through a $k$-tuple $\Sigma$ of $\sigma_{k}$ and, for given $X, Y$ runs through an $l$-tuple $\mathrm{T}_{X}$ of $\tau_{l}$. For example, if $S=T$ is the space of real numbers modulo 1 and $\sigma_{2}(\alpha)$ the class of pairs $x, x^{\prime}$ having $x-x^{\prime}=\alpha$, then $\sigma_{4}(\alpha, \beta)=\sigma_{2}(\alpha) \times \sigma_{2}(\beta)$ consists of quadruples $(x, y)$, $(x, y+\beta),(x+\alpha, \bar{y}),(x+\alpha, \bar{y}+\beta)$.

Theorem 2. Assume $\sigma_{k}$ has property $P$ in $S, \tau_{l}$ has property $P$ in $T$ and $\tau_{l}$ is closed. Then $\sigma \times \tau$ has property $P$ in $S \times T$.

It appears to be difficult to generalize our results to maps $f$ into $R^{n}$ and to prove the following generalization of the Borsuk-Ulam Theorem: Let $X \rightarrow-X$ be the antipodal map in $S^{n}$ and let $f$ be a map of $S^{n} \times S^{n}$ into $R^{n}$. Then there exist $X, Y, \bar{Y}$ in $S^{n}$ having $f(X, Y)$ $=f(X,-Y)=f(-X, \bar{Y})=f(-X,-\bar{Y})$.

\section{The proofs.}

Lemma 1. A ssume $R$ is a roof over $S \times T$ and let $x(t), X(t)$ be a rain $N$ over $S$. Then the set $G(N)$ of points $(t, Y)$ of $C(T)$ where

$$
(x(t), X(t), Y) \in R
$$

forms a roof over $T$.

Proof. If $\left(t_{n}, Y_{n}\right)$ is a sequence in $G(N)$, then $\left(x\left(t_{n}\right), X\left(t_{n}\right), Y_{n}\right) \in R$ has a subsequence convergent to some $(x, X, Y) \in R$. For this subsequence $x\left(t_{n}\right)$, and therefore $t_{n}$, is bounded, and $t_{n}$ will have a limitpoint $t_{0}$ where $x=x\left(t_{0}\right), X=X\left(t_{0}\right)$. Thus $\left(t_{0}, Y\right)$ will be a limit-point of $\left(t_{n}, Y_{n}\right)$ in $G(N)$, and $G(N)$ is compact.

Thus if $G(N)$ were not a roof, there would exist a rain $t(s), Y(s)$ over $T$, having no point in $G$. Then $x(t(s)), X(t(s)), Y(s)$ would be a rain over $S \times T$ with no point in $R$.

LEMMA 2. Let $R$ be a roof over $S \times T$ and assume $\tau_{l}$ of $T$ is closed and has property $P$. Then the set $H$ of points $(x, X)$ in $C(S)$ such that for suitable $\mathrm{T}(x, X) \in \tau$ 


$$
(x, X, Y) \in R \text { for every } Y \in \mathrm{T}
$$

is a roof over $S$.

Proof. By $R^{(l)}$ denote the set of points $\left(x, X, Y_{1}, \cdots, Y_{l}\right)$ of $C(S \times T \times \cdots \times T)$ such that $\left(x, X, Y_{j}\right) \in R(j=1, \cdots, l)$ and $Y_{1}, \cdots, Y_{l}$ is an $l$ tuple of $\tau_{l}$. It follows from the compactness of $R$ and the closedness of $\tau_{l}$ that $R^{(l)}$ is compact. $(x, X)$ is in $H$ if and only if there exist $Y_{1}, \cdots, Y_{l}$ with $\left(x, X, Y_{1}, \cdots, Y_{l}\right) \in R^{(l)}$. Therefore $H$ is compact.

Now let $N$ be a rain over $S$. Then $G(N)$ is a roof over $T$ and there exists some $t$ and some $\mathrm{T} \in \tau$ such that $(t, Y) \in G$ for every $Y \in \mathrm{T}$. Then $(x(t), X(t), Y) \in R$ for every $Y \in \mathrm{T}$ and $N$ has a common point with $H$.

Proof of Theorem 2. Assume the hypotheses of the theorem to be satisfied. Construct $H$ as in Lemma 2. By the property of $\sigma$, there exists a $\Sigma \in \sigma$ and some $x$ such that

$$
(x, X) \in H \text { for every } X \in \Sigma .
$$

Then $(x, X, Y) \in R$ for $X \in \Sigma, Y \in \mathrm{T}_{X}$ and Theorem 2 is proved.

ProOF OF TheOREM 1 . If $S$ is the space of real numbers modulo 1 and $\sigma_{2}(\alpha)$ is defined as before, then $\sigma_{2}(\alpha)$ has property $P$. This is the one-dimensional case of the generalized Yamabe-Yujobo Theorem. Furthermore, $\sigma_{2}$ is closed. Theorem 1 is a consequence of these facts and Theorem 2.

\section{REFERENCES}

1. W. Schmidt, Stetige Funktionen auf dem Torus, J. Reine Angew. Math. vol. 207 (1961) pp. 86-95.

2. H. Yamabe and Z. Yujobo, On the continuous functions defined on a sphere, Osaka Math. J. vol. 2 (1950) pp. 19-22.

University of Colorado 